\title{
The implementation of randomized trials in the treatment of advanced ovarian cancer with HIPEC: waiting for new evidence.
}

\author{
Coccolini $\mathrm{F}^{1}$, , Lotti $\mathrm{M}^{1}$, Catena $\mathrm{F}^{2}$, Pisano $\mathrm{M}^{1}$, Grosso $\mathrm{G}^{3}$, Frigerio $\mathrm{L}^{3}$ and Ansaloni $\mathrm{L}^{1}$ \\ ${ }^{1}$ General and Emergency surgery department, OspedaliRiuniti, Bergamo, Italy \\ ${ }^{2}$ General and Emergency surgery department, Ospedale Maggiore, Parma, Italy \\ ${ }^{3}$ Obstetrics and Gynecology department, OspedaliRiuniti, Bergamo, Italy
}

\begin{abstract}
Ovarian cancer (OC) is one of the most frequent mortality causes among female neoplasms. A lot of data is available on the efficiency and the relative safety of the different treatments. In the last twenty years the overall survival (OS) and the disease free survival (DFS) have enormously incremented thanks to the combined chemotherapy regimens and the incredible progresses of surgery. These progresses are also related to the increased ability in managing patients more and more compromised, i.e. older and weaker. In addition to that, more than twenty years ago, Sugarbaker et al. started to treat the peritoneal cancer diffusion as a loco-regional disease. This change of practice has been demonstrated to be a real revolution by following studies.
\end{abstract}

Keywords: randomized trial; HIPEC; ovarian cancer, carcinosis; quality of life

The hyperthermicintraperitoneal chemotherapy (HIPEC) proved its capability to prolong both OS and DFS in many other different pathologies such as colon and gastric cancer, pesudomixomaperitonei and peritoneal mesothelioma [1]. Many phase I and II studies in the use of HIPEC in OC showed that it is related to positive results and an acceptable morbidity rate [2]. For this reason the time has come for randomized trials (RT) in order to determinate the real effect of HIPEC in OC. At present we know of 7 RT evaluating the effectiveness of HIPEC in primary or recurrent OC. They are at different stages: two have only been proposed while five are already on course[3-8].

The two proposed ones aim to investigate HIPEC in primary and recurrent OC [8]. They scheduled to randomize separately primary and recurrent cancers in two different groups. Both arms would then receive complete cytoreductive surgical procedures either followed by HIPEC or not.

Among the ongoing trials the first one is a Korean study (NCT01091636) [5]. It evaluates the efficacy of HIPEC in the treatment of primary and recurrent OC. The second one is a study from The Netherlands (NCT00426257) [4]. It is a phase III RT on the efficacy of HIPEC after secondary debulking surgery. The two remaining trials study its efficacyin recurrentOC. The first one is a multicentric phase III trial from France: the CHIPOR study (NCT01376752) [6]. The second one is an Italian multicentric phase III trial: the HORSE study (NCT01539785) [7]. The last published protocol evaluating HIPEC in primary advanced OC (NCT01628380) [3] is the CHORINE study. This is an Italian multicentric trial on the role of HIPEC after chemotherapy in those patients who responded to the treatment.

It's very difficult to evaluate the real impact of HIPEC in terms of OS and DFS. Too much bias can interfere with the final results. That is why in HIPEC standardized criteria

*Corresponding author: Coccolini F, Unit of General and Emergency surgery, OspedaliRiuniti, Largo Barozzi 1, 24128 Bergamo, Italy, Tel.: +39 352 66515; Fax: +3935266567; E-mail: federico.coccolini@gmail. com

Received 11 November 2012 Revised 29 December 2013 Accepted 5 January 2013 Published 12 January 2013

Citation: Coccolini F, Lotti M, Catena F, Pisano M, Grosso G, Frigerio L, Ansaloni L (2013) The implementation of randomized trials in the treatment of advanced ovarian cancer with HIPEC: waiting for new evidence. J Cancer Res Ther. 1: 60-61. doi:10.14312/20524994.2013-9

Copyright: (C) 2013 Coccolini F, et al. This is an open-access article distributed under the terms of the Creative Commons Attribution License, which permits unrestricted use, distribution and reproduction in any medium, provided the original author and source are credited. 
have not been adopted yet. Each protocol applies different methods: different drug doses, different perfusion times at different temperatures, different criteria to evaluate completeness of cytoreduction and different classifications for complications. Moreover the randomization is planned at different time points. Added to that only a few evaluate the drug dosage in peritoneal fluid, plasma and tissues establish the efficiency of the intra-abdominal perfusion. Many centres treat each year a small number of patients and that's why they lack the necessary surgical expertise in treating advanced and peritoneal spread cancer.

As a counterpartpublished data areabsolutely encouraging and demonstrate that the scientific community is on the right path. Nonetheless a great effort has still to be done to unify the protocols and to increase the number of women treated homogeneously. In our opinion in a few years this will lead to significant results. Patients need to be centralized to the higher experienced centres to allow them to participate in the ongoing trials. Centralization could also give the patients the possibility to undergo a real complete cytoreduction without the risk of residual disease. This has been demonstrated to have a positive impact on survival rates.

Last but not least we would like to stress the importance of taking into consideration the quality of life in treating patients. This will give a real chance to choose between different treatments with a realistic prospective on the post-treatment life.

\section{Conflict of Interest}

The authors wish to express that they have no conflict of interest.

\section{References}

[1] Roviello F, Caruso S, Marrelli D, Pedrazzani C, Neri A, et al. (2011) Treatment of peritoneal carcinomatosis with cytoreductive surgery and hyperthermicintraperitoneal chemotherapy: state of the art and future developments. SurgOncol 20:e38-e54.

[2] Mulier S, Claes JP, Dierieck V, Amiel JO, PahautJP, et al. (2012) Survival benefit of adding HyperthermicIntraPEritoneal Chemotherapy (HIPEC) at the different time-points of treatment of ovarian cancer: review of evidence. Curr Pharm Des 18:3793-3803.

[3] www.clinicaltrials.gov/ct2/show/record/NCT01628380

[4] www.clinicaltrials.gov/ct2/show/record/NCT00426257

[5] www.clinicaltrials.gov/ct2/show/record/NCT01091636

[6] www.clinicaltrials.gov/ct2/show/record/NCT01376752

[7] www.clinicaltrials.gov/ct2/show/record/NCT01539785

[8] Chua TC, Liauw W, Robertson G, Morris DL (2009) Establishing evidence for change in ovarian cancer surgery--proposing clinical trials of cytoreductive surgery and hyperthermicintraperitoneal chemotherapy (HIPEC) in ovarian cancer peritoneal carcinomatosis. GynecolOncol 115:166-168. 